\title{
White Matter, Gray Matter and Cerebrospinal Fluid Segmenta- tion from Brain 3D MRI using B-UNET
}

\author{
Tran Anh Tuan ${ }^{\mathrm{a},{ }^{*}}$, Pham The Bao ${ }^{\mathrm{b}}$, Jin Young Kim ${ }^{\mathrm{c}}$, João Manuel R. S. Tavares ${ }^{\mathrm{d}}$ \\ ${ }^{a}$ Faculty of Mathematics and Computer Science, University of Science, Vietnam National University, Ho \\ Chi Minh City, Vietnam \\ ${ }^{b}$ Department of Computer Science, Sai Gon University, Vietnam \\ ${ }^{c}$ Department of Electronic and Computer Engineering, Chonnam National University, South Korea \\ ${ }^{d}$ Instituto de Ciência e Inovação em Engenharia Mecânica e Engenharia Industrial, Departamento de En- \\ genharia Mecânica Faculdade de Engenharia, Universidade do Porto, Portugal
}

*Corresponding author: tatuan@hcmus.edu.vn

\begin{abstract}
The accurate segmentation of brain tissues in Magnetic Resonance (MR) images is an important step for detection and treatment planning of brain diseases. Among other brain tissues, Gray Matter, White Matter and Cerebrospinal Fluid are commonly segmented for Alzheimer diagnosis purpose. Therefore, different algorithms for segmenting these tissues in MR image scans have been proposed over the years. Nowadays, with the trend of deep learning, many methods are trained to learn important features and extract information from the data leading to very promising segmentation results. In this work, we propose an effective approach to segment three tissues in 3D Brain MR images based on B-UNET. The method is implemented by using the Bitplane method in each convolution of the UNET model. We evaluated the proposed method using two public databases with very promising results.
\end{abstract}

Keywords: Image segmentation, Medical Imaging, Deep Learning, Bitplane-UNET

\section{Introduction}

In our aging society, many degenerative diseases of the nervous system are becoming more and more common. Neurodegenerative diseases represent a large group of neurological disorders with heterogeneous clinical and pathological effects on specific subsets of neurons in specific functional anatomic systems [1]. Many symptoms related to these diseases can be detected by using medical imaging. The most common imaging technique for the examination of brain diseases is Magnetic Resonance Imaging (MRI), which allows the visualization of the structure of the brain tissues. However, a crucial step in the diagnosis and treatment of brain diseases based on MRI is the accurate segmentation of the imaged brain tissues.

Many methods have been proposed for brain tissues segmentation from 3D Brain MRI [2-4]. Among other brain tissues, Gray Matter, White Matter, and Cerebrospinal Fluid are usually segmented for Alzheimer diagnosis purpose [5-8]. In the group of machine learning networks, Convolutional Neural Network (CNNs) has been successfully used to segment and classify medical images for many years $[9,10]$. A CNN uses layers to transform the input data trough 
filters in order to obtain automatically the features for classification. There are recent approaches using CNNs for image segmentation that have shown better results than the previous ones such as UNET [11]

This article is organized in four sections. Section 2 presents the proposed method, which is based on Bitplane and UNET. Section 3 reports the experiments using two image databases: IBSR and MICCAI challenge 2018 datasets. The conclusions are presented in the last section.

\section{Proposed method}

The proposed method is composed of two main steps: Skull stripping and Brain tissues segmentation, Figure 1. From the original 3D MR image, we tranform the 3D dataset into 2D slices, and implement UNET model for the image segmentation based on 2 steps, which is an extension of each neuronal convolution using features generating from the Bitplane method.

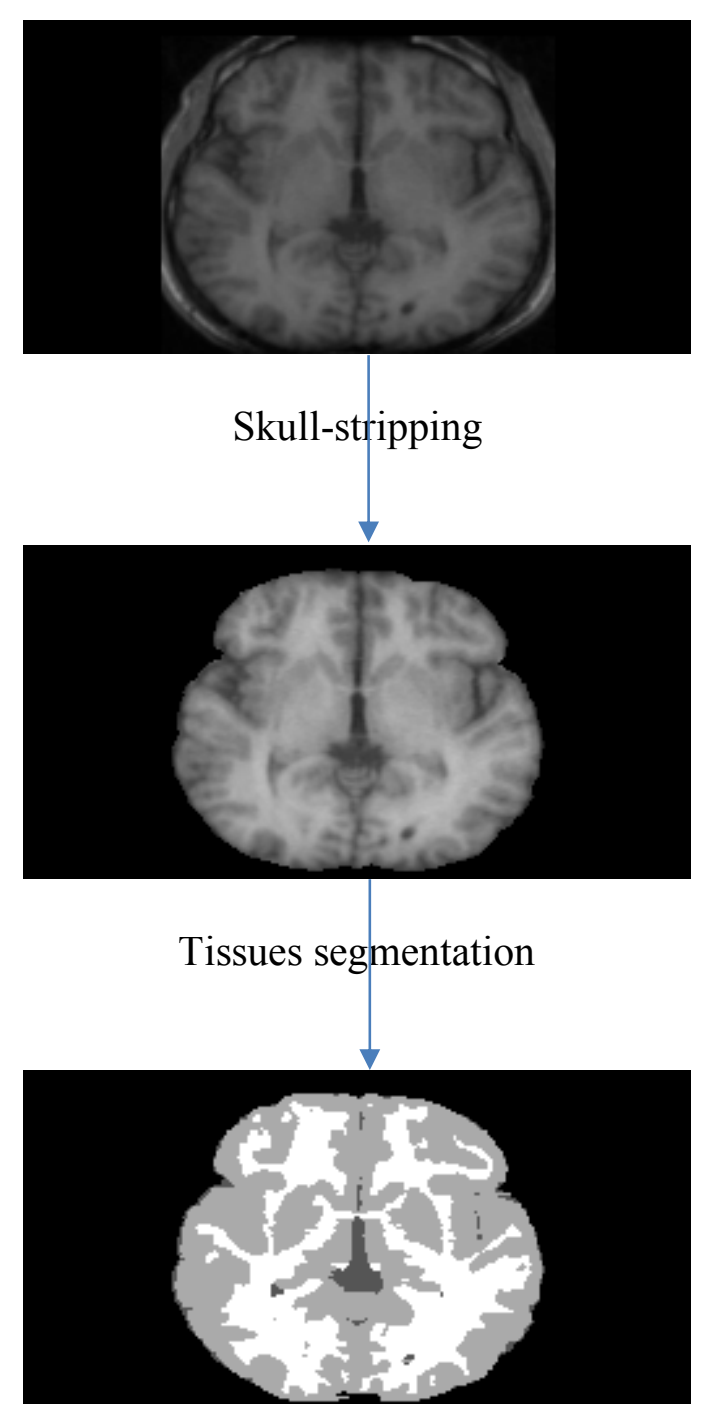

Fig. 1. The overview of the proposed method to segment three brain tissues in 3D MR images. 
The Bitplane method [12] is based on decomposing a multilevel image into a series of binary images. The intensities of an image are based on:

$a_{m-1} 2^{m-1}+a_{m-2} 2^{m-2}+\cdots+a_{1} 2^{1}+a_{0} 2^{0}$

where $a$ is the order bit of each pixel and $m$ is the grey level in the input image.

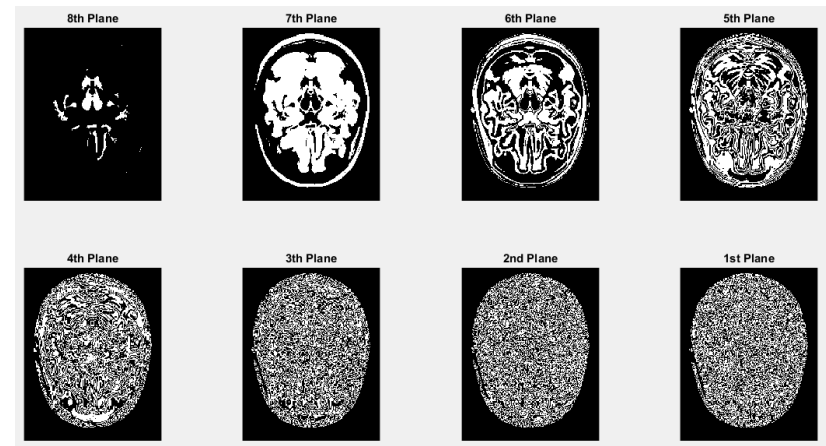

Fig. 2. Example of eight-bit planes of an image [13].

We represented each input image slice by eight-bit planes as shown in Fig. 2. We realized that higher-order bits contain most of the significant commonly used visual information, and that lowerorder bits contain subtle details that are not clinically relevant. In some cases, in order to reduce the effect of small intensity variations, we can use the OR operation between two bit planes: $(\mathrm{m}-1)^{\text {th }}$ bit and $(\mathrm{m}-2)^{\text {th }}$ bit planes:

$g_{i}=a_{m-1} \oplus a_{m-2}$

One of the CNN models commonly used in medical image segmentation is UNET [11], which is based on autoencoder. Here, we propose an approach by extending each convolution in the UNET model. With the input of convolution, multiple different features are generated based on the bit values of each pixel of the image, by using the Bitplane method, and the output is the convolution of the combination of these features. Let's consider layer $i$, and $L=\left\{K_{1}, \ldots, K_{n}\right\}$ as the set of output by using the Bitplane method. Hence, the number of feature maps that layer $i$ generates is equal to the merge of the number of feature maps that the layer $i$ generates. For example, as shown in Figure 3, instead of using only convolution for input, multiple binary features are generated after using convolution and the output is the merge as Add operator for these binary features. 


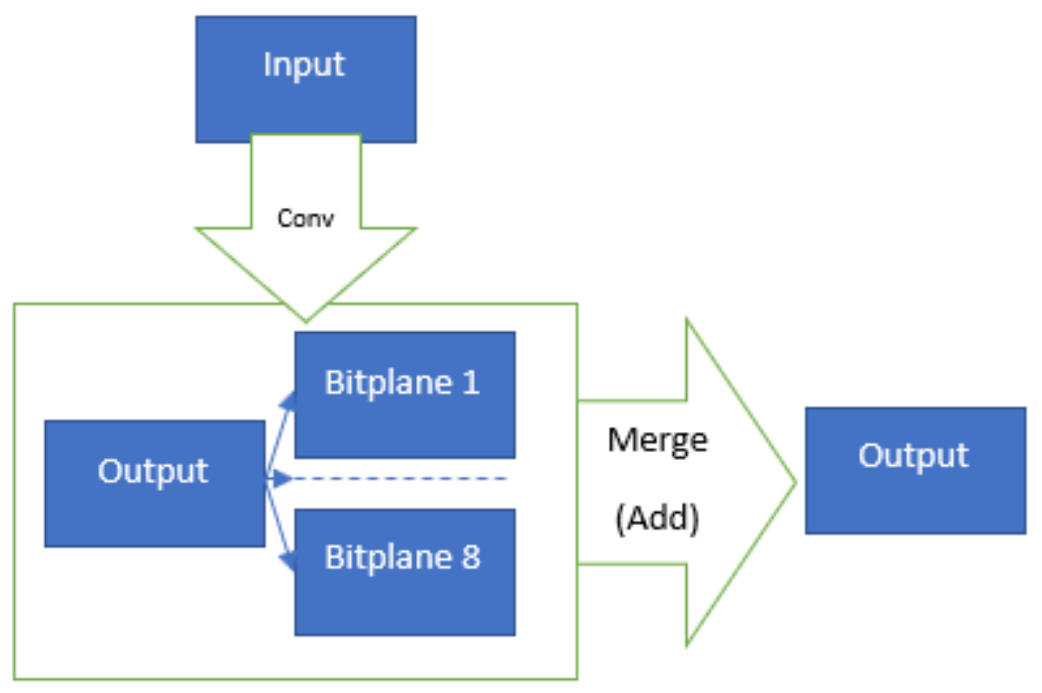

Fig. 3. Example of using the Bitplane method with a convolution.

\section{Experiments and Discussion}

We evaluated the proposed method on the IBSR 18 image database [14], which contains real cases and has been used in many studies regarding brain tissues segmentation in MR image scans. We also evaluated the method with a database from a MICCAI challenge [15] that is often used for comparing methods for the segmentation of gray matter, white matter, cerebrospinal fluid, and other structures on 3T MR image scans of the brain, and to assess the effect of severe pathologies on the segmentation results and brain volumetry. One of the most common metrics used for evaluating the performance of a segmentation method is Dice Similarity Coefficient [16]:

$$
S(X, Y)=\frac{2|X \cap Y|}{|X|+|Y|}
$$

where $|X|$ and $|Y|$ are the number of non zero voxels in sets $X$ and $Y$, and $|X \cap Y|$ is the number of non zero voxels shared by the two sets, respectively.

IBSR 18 database: This image dataset is composed of $18 \mathrm{~T} 1$ weighted MRI scans with $1.5 \mathrm{~mm}$ of slice thickness $(256 \times 128 \times 256)$. These volumes are provided after skull-stripping, normalization and bias field correction. The provided ground truth is composed of manual segmentations performed by experts using tissue labels as 0, 1, 2, 3 for background, CSF, GM, and WM, respectively. Each MRI brain volume has 128 slices of size $256 \times 256$ pixels each.

Our proposed method was implemented based on the Keras library [17] with back-end Tensorflow [18] supporting convolutional network. The used optimizer was 'Adam' [19] and the loss was selected as 'binary_crossentropy' loss [20]. We evaluated the IBSR 18 database with k-folds $=3$ and the obtained results are shown in Table 1. An example of a segmentation result is shown in Figure 4.

Table 1 - The Dice values obtained for the IBSR 18 dataset.

\begin{tabular}{|c|c|c|c|c|}
\hline & WM & GM & CSF & Average \\
\hline U-NET & 0.92 & 0.92 & 0.77 & 0.87 \\
\hline B-UNET & $\mathbf{0 . 9 3}$ & 0.92 & $\mathbf{0 . 7 8}$ & $\mathbf{0 . 8 8}$ \\
\hline
\end{tabular}



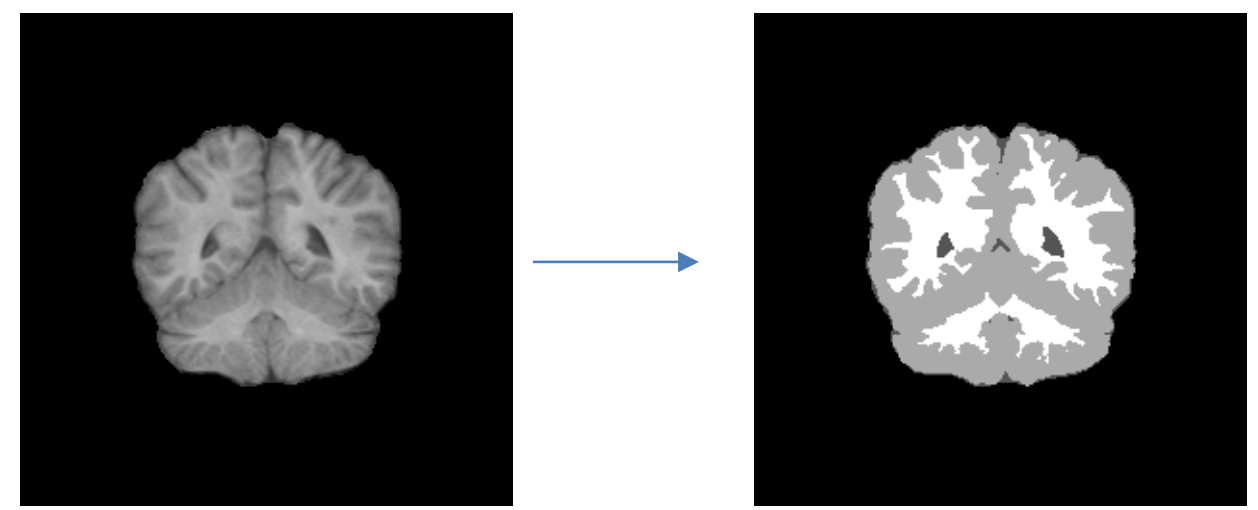

Fig 4. Example of a segmentation result in the IBSR dataset (slice: 40 - person IBSR_01).

The Database Grand Challenge on MR Brain Segmentation proposed in MICCAI 2018 consists of 7 sets of MR brain images (T1, T1 inversion recovery, and T2-FLAIR), which are accompanied with the manual segmentation of three brain structures made by experts. All image scans have a voxel size of $0.958 \mathrm{~mm} \times 0.958 \mathrm{~mm} \times 3.0 \mathrm{~mm}$ and all are already aligned and biased field corrected using the N4ITK algorithm [21]. Here, we only used T1 and T2-FLAIR images as input for segmentation.

Our method was implemented based on a Keras library with back-end Tensorflow supporting convolutional network. We evaluated the database with $\mathrm{k}$-folds $=3$ obtaining the results indicated in Table 2. T. An example of a segmented image of this dataset is shown in Figure 5.

Table 2. The Dice values obtained for MICCAI 2018 MRBRAIN dataset.

\begin{tabular}{|c|c|c|c|c|}
\hline & WM & GM & CSF & Average \\
\hline U-NET & 0.87 & 0.82 & 0.87 & 0.85 \\
\hline B-UNET & 0.87 & $\mathbf{0 . 8 3}$ & $\mathbf{0 . 8 8}$ & $\mathbf{0 . 8 6}$ \\
\hline
\end{tabular}
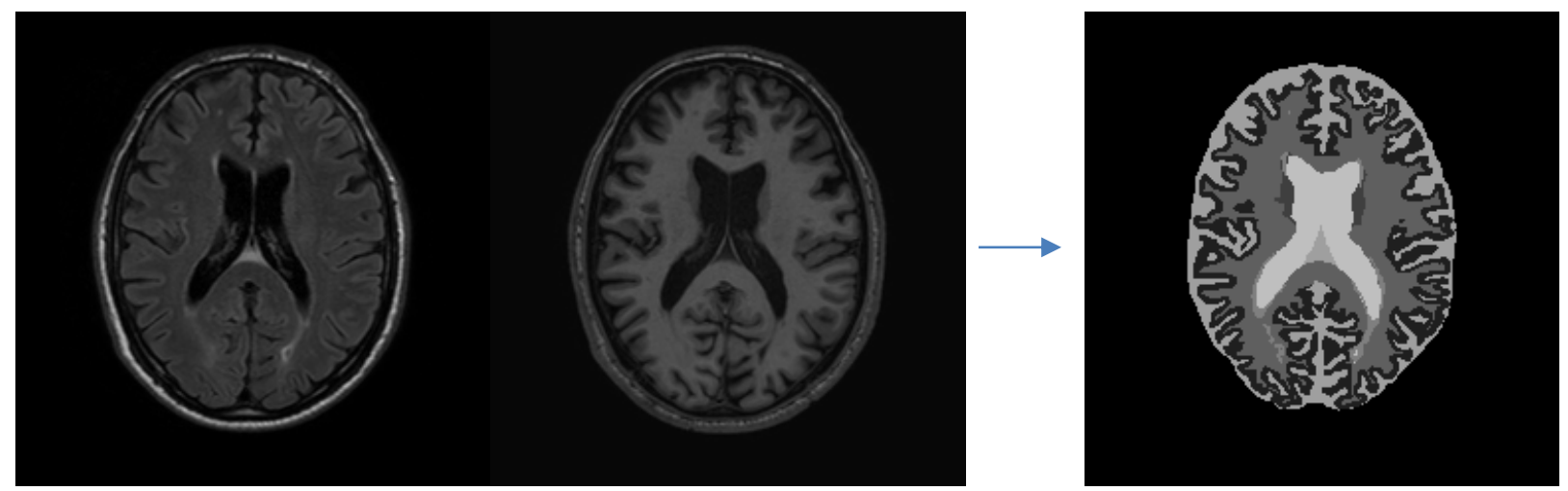

Fig 5. Example of a segmentation result in the MRBRAIN MICCAI 2018 dataset (slice 25 person $1)$. 
From the results obtained in the two evaluations done, we can conclude that very posimissing results were achivied as to the segmentation of the brain tissues. In our approach, we extended a well known architecture for 2D image segmentation (UNET) by replacing 2D convolutions with Bitplane Convolutions. From each convolution, one feature is extended multiple features that come from bit positions. The important and hidden features combined with features generated using the UNET model allows the achivement of superior segmentation results.

With our approach, the segmenation results are better thand the ones obtained the original UNET because in each convolution, the original convolution method is combined with the convolution of other features to get the best classification features. However, this has high computational cost and, therefore, it imrpoved model is slower to train than the original UNET model.

\section{Conclusion}

In this study, we proposed an approach to segment brain tissues in 3D MR images. The proposed solution is based on the building of more features and their combination in each convolution. Here, we implemented to generate multiple binary features by using Bitplane method in each convolution of the UNET model. The proposed method was evaluated using two public databases and obtained very promising results, basically, because the original input of each convolution is generated into multiple features before using convolution. In the future, we will concentrate on the extension of other operators in the classification model to get even better segmentation results.

\section{Acknowledgments}

This work was supported by Erasmus Mundus Program, reference IMPAKT Project 2017-2018.

João Manuel R.S. Tavares gratefully acknowledges the funding of Project NORTE-01-0145FEDER-000022 - SciTech - Science and Technology for Competitive and Sustainable Industries, cofinanced by "Programa Operacional Regional do Norte" (NORTE2020), through "Fundo Europeu de Desenvolvimento Regional" (FEDER).

\section{References}

[1] Serge Przedborski, Miquel Vila, Vernice Jackson-Lewis, Series Introduction: Neurodegeneration: What is it and where are we?, J Clin Invest. 2003; 111(1): 3-10, doi: 10.1172/JCI200317522

[2] L. Dora, S. Agrawal, R. Panda, and A. Abraham, State of the art methods for brain tissue segmentation: A review, IEEE Rev Biomed Eng. 2017;10:235-249, doi: 10.1109/RBME.2017.2715350 [3] Zeynettin Akkus, Alfiia Galimzianova, Assaf Hoogi, Daniel L. Rubin, Bradley J. Erickson, Deep Learning for Brain MRI Segmentation: State of the Art and Future Directions, J Digit Imaging. 2017; 30(4): 449-459, doi: 10.1007/s10278-017-9983-4

[4] Princi Soni, Vijayshri Chaurasia, MRI Segmentation for Computer-Aided Diagnosis of Brain Tumor: A Review, Machine Intelligence and Signal Analysis. Advances in Intelligent Systems and Computing, 2018, 748:375-38, doi: 10.1007/978-981-13-0923-6_33

[5] Sandhya Gudise, Giri Babu Kande, Satya Savithri T, Segmentation of MR Images of the Brain to Detect WM, GM, and CSF Tissues in the Presence of Noise and Intensity Inhomogeneity, IETE Journal of Research, 2019, 65(2): 250-262, doi: 10.1080/03772063.2017.1409088 
[6] Wang Y, Wang Y, Zhang Z, Xiong Y, Zhang Q, Yuan C, Guo H, Segmentation of gray matter, white matter, and CSF with fluid and white matter suppression using MP2RAGE, J Magn Reson Imaging. 2018;48(6):1540-1550, doi: 10.1002/jmri.26014

[7] Andrei Irimia, Alexander S. Maher, Kenneth A. Rostowsky, Nahian F. Chowdhury, Darryl H. Hwang, E. Meng Law, Brain Segmentation From Computed Tomography of Healthy Aging and Geriatric Concussion at Variable Spatial Resolutions, Front. Neuroinform, 2019, 13:9, doi: 10.3389/fninf.2019.00009

[8] Illan IA, Górriz JM, Ramírez J, Meyer-Base A, Spatial component analysis of MRI data for Alzheimer's disease diagnosis: a Bayesian network approach, Front. Comput. Neurosci., 2014, 8:156, doi: 10.3389/fncom.2014.00156

[9] Xiaolong Liu, Zhidong Deng, Yuhan Yang, Recent progress in semantic image segmentation, Artificial Intelligence Review, 2019, 52(2):1089-1106, doi:10.1007/s10462-018-9641-3

[10] Yanming Guo, Yu Liu, Theodoros Georgiou, Michael S. Lew, A review of semantic segmentation using deep neural networks International Journal of Multimedia Information Retrieval, International Journal of Multimedia Information Retrieval, 2018, 7(2):87-93, doi: 10.1007/s13735-017$0141-\mathrm{z}$

[11] Olaf Ronneberger, Philipp Fischer, Thomas Brox. U-Net: Convolutional Networks for Biomedical Image Segmentation. Medical Image Computing and Computer-Assisted Intervention (MICCAI), Springer, 2015, 9351: 234-241, doi: 10.1007/978-3-319-24574-4_28

[12] R.C. Gonzalez, R.E. Woods, Digital Image Processing. Prentice Hall, Inc., New Jersey, 2002.

[13] Tuan T.A., Kim J.Y., Bao P.T., 3D brain magnetic resonance imaging segmentation by using bitplane and adaptive fast marching, Int. J. Imaging Syst. Technol, 2018, 28: 223-230, doi:10.1002/ima.22273

[14] Frazier, JA, et al., Internet Brain Segmentation Repository (IBSR) $1.5 \mathrm{~mm}$ dataset, Child and Adolescent Neuro Development Initiative, 2007

[15] https://mrbrains 18.isi.uu.nl, 2018, last access 05/2019

[16] L. R. Dice, Measures of the amount of ecologic association between species, Ecology. 26(3)(1945):297-302, doi: 10.2307/1932409

[17] Chollet, Francois and others, Keras. https://keras.io, 2015, last access 05/2019

[18] Martín Abadi, Ashish Agarwal, et al., TensorFlow: Large-scale machine learning on heterogeneous systems, 2015, last access 05/2019

[19] D.P. Kingma, L.J.Ba, Adam: a Method for Stochastic Optimization, in: International Conference on Learning Representations, California, 2015, arXiv :1412.6980

[20] Bishop, Christopher M., Pattern Recognition and Machine Learning, Springer, 2006

[21] Nicholas J. Tustison, Brian B. Avants, Philip A. Cook, Yuanjie Zheng, Alexander Egan, Paul A. Yushkevich, James C. Gee, N4ITK: Improved N3 Bias Correction, IEEE Trans Med Imaging, 2010, 29(6): 1310-1320, doi: 10.1109/TMI.2010.2046908 